\title{
A Multimodal Nonlinear Tuned Vibration Absorber
}

\author{
Ghislain Raze and Gaetan Kerschen
}

\begin{abstract}
This paper presents a tuning methodology to design a vibration absorber able to mitigate the vibratory amplitude of multiple resonances of a nonlinear structure. The linear characteristics of the absorber are first tuned to obtain the equal-peak design on every mode to be controlled when the structure is behaving linearly. Nonlinearities are then introduced intentionally in the absorber to counteract the effect of the nonlinearities inside the host structure. Their functional form is chosen according to a principle of similarity, and their coefficients are determined to enforce equal peaks in the nonlinear regime.
\end{abstract}

\section{Introduction}

Engineering structures are becoming lighter and more complex to suit the needs of an ever-increasing demand for performance and to comply with stringent regulations. This trend comes with several challenges, one of which being the increased susceptibility to high-amplitude vibrations. These vibrations can be detrimental to the device performance and lifetime, or even be threatening safety. Passive vibration reduction techniques can provide a solution to this issue. The linear tuned vibration absorber (LTVA, also often referred to as "tuned mass damper" or "dynamic vibration absorber") enters this category and is a widely-used device of proven efficiency [4].

The LTVA was first proposed by Frahm [5]. By attaching a one-degree-of-freedom undamped oscillator to a host structure, one particular vibration mode can be completely suppressed. Ormondroyd and den Hartog [11] proposed to add a damping

G. Raze

Space Structures and Systems Laboratory, Allée de la Découverte 9, Liege B-4000, Belgium, email: g.raze@uliege.be

G. Kerschen

Space Structures and Systems Laboratory, Allée de la Découverte 9, Liege B-4000, Belgium, e-mail: g.kerschen@uliege.be 
element to broaden the bandwidth in which the absorber is efficient. They based the tuning methodology on invariant points in the frequency response function. These points are insensitive to the absorber damping. The stiffness of the absorber is tuned to make the amplitude of the frequency response function at these points equal and the absorber damping may be chosen so that one of this two points is a local maximum of the frequency response function, yielding two possible values. Brock [1] later proposed to take the average of these damping values to yield a nearly optimal design. Almost sixty years later, Nishihara and Asami [10] found the exact closedform solution to this problem, based on the minimization of the maximal vibratory amplitude of the host structure. With this design, the frequency response function of the controlled structure exhibits two peaks of equal amplitude near the original resonance of the host structure. Hence, this design was termed equal-peak design.

Many structures might have multiple resonances inside a frequency band of interest. Considering a structure with multiple modes brings new challenge to the design of tuned vibration absorbers. The influence of non-resonant modes might detune the absorber, which is detrimental for its performance. Krenk and Høgsberg [9] proposed to introduce quasi-dynamic background corrections to take into account the influence of non-resonant modes. Ozer and Royston [12] proposed a numerical optimization algorithm based on the invariant points of the frequency response function to tune the absorbers. If the excitation frequency is uncertain or varying, or the structure is subjected to a multiharmonic or broadband forcing, multiple resonances might be excited. A simultaneous control of these resonances may be desirable.

Lighter and more flexible structures are more prone to high-amplitude vibrations. These vibrations can trigger the nonlinearities present in the structure. One peculiarity of nonlinear structures is their frequency-energy dependence: the resonance frequencies of a nonlinear structure may change with the forcing amplitude. This can be particularly detrimental to tuned vibration absorbers, as the nonlinear resonance frequencies shift away from their initial position, detuning the absorber. This detuning is often the cause for a loss of performance. Habib and Kerschen [8] purposely used a nonlinear stiffness in a nonlinear tuned vibration absorber (NLTVA) to counteract this undesirable phenomenon. They proposed to use a principle of similarity for the design of nonlinear vibration absorbers. This principle states that the functional form of the nonlinearity in the absorber should be identical to that of the host structure. They designed a nonlinear vibration absorber able to maintain equal peaks over a broader range of forcing amplitude than when using a linear vibration absorber. This same principle of similarity was recently used by Habib and Kerschen [7] to modify the characteristics of multiple nonlinear resonances. By introducing and properly tuning nonlinearities in a structure, they were able to linearise its dynamics.

The purpose of this work is to associate the multimodal and nonlinear aspects of a tuned vibration absorber. More specifically, this paper describes how to design a vibration absorber able to mitigate the vibratory amplitude of multiple nonlinear resonances. To this end, the absorber is first designed considering the underlying linear dynamics of the host structure. A design approach based on a modal expansion of the frequency response function of the host structure is proposed. If more preci- 
sion is required in the tuning, an objective function for an optimisation algorithm is proposed. The nonlinear behaviour of the structure is then taken into account. Nonlinearities are intentionally introduced in the absorber. Their functional form is chosen according to a principle of similarity. The same formalism as [7] is used to impose equal peaks in the nonlinear frequency response function. With this new design, the working range of vibration absorbers can be extended to higher forcing amplitudes.

\section{Multimodal Linear Tuned Vibration Absorber}

This section introduces a tuning methodology for multiple LTVAs placed on an undamped structure. The structure is modelled as a one-degree-of-freedom oscillator from the point of view of the absorber, similarly to [9]. With this model, it is possible to tune the parameters of the absorber. If a further precision is required, a cost function for an optimisation algorithm is proposed.

The undamped equations of motion of the host structure are given by

$$
\mathbf{M}_{0} \ddot{\mathbf{x}}_{0}+\mathbf{K}_{0} \mathbf{x}_{0}=\mathbf{f}_{0}(t)
$$

where $\mathbf{M}_{0}$ is the structural mass matrix, $\mathbf{K}_{0}$ is the structural stiffness matrix, $\mathbf{x}_{0}$ is the vector of generalized coordinates and $\mathbf{f}_{0}(t)$ is the generalized loading vector. The subscript 0 indicates quantities exclusively related to the host structure. Under the assumption of a periodic forcing, i. e. $\mathbf{f}_{0}(t)=\mathbf{f}_{0} \cos \omega t$, the equations of motion can be solved by expanding the response in the basis of the structural normal modes and by projecting them onto this basis [6]. Eventually, by reconstructing the physical response from the modal response,

$$
\mathbf{x}_{0}=\boldsymbol{\Phi}_{0}\left(\boldsymbol{\Omega}_{0}^{2}-\omega^{2} \mathbf{I}\right)^{-1} \boldsymbol{\Phi}_{0}^{T} \mathbf{f}_{0}
$$

where $\boldsymbol{\Phi}_{0}$ is the matrix of mass-normalized mode shapes, $\boldsymbol{\Omega}_{0}$ is a diagonal matrix containing the associated mode frequencies, $\mathbf{I}$ is the identity matrix and the superscript $T$ denotes a transposition. This harmonic response can be used in the design of the absorbers. Assuming that the absorber is placed at a location given by the localisation vector $\mathbf{w}$, its base displacement can be found as $u=\mathbf{w}^{T} \mathbf{x}_{0}$. Introducing

$$
\begin{gathered}
\mathbf{w}^{T} \boldsymbol{\Phi}_{0}=\left[\phi_{u, 1}, \cdots, \phi_{u, N_{0}}\right], \\
\boldsymbol{\Phi}_{0}^{T} \mathbf{f}_{0}=\left[\phi_{f, 1}, \cdots, \phi_{f, N_{0}}\right]^{T} f_{0},
\end{gathered}
$$

with the number of modes $N_{0}$ and the forcing amplitude $f_{0}$, the forced harmonic response can be expressed as a sum of contributions from the different modes 


$$
u=\sum_{i=1}^{N_{0}} \frac{\phi_{u, i} \phi_{f, i}}{\omega_{i}^{2}-\omega^{2}} f_{0} .
$$

Around the resonance frequency of mode $i$, the harmonic response may be approximated by that of a single-degree-of-freedom spring-mass system. Neglecting all the terms different than those related to mode $i$ in the sum of Equation (5), the modal mass $m_{i}$ and the modal stiffness $k_{i}$ are identified as

$$
m_{i}=\frac{1}{\phi_{u, i} \phi_{f, i}}, \quad k_{i}=\frac{\omega_{i}^{2}}{\phi_{u, i} \phi_{f, i}},
$$

respectively. Using this one-degree-of-freedom approximation, the stiffness $k_{a, i}$ and damping $c_{a, i}$ of an LTVA of mass $m_{a, i}$ designed to mitigate the vibrations of mode $i$ can be determined using the classical one-degree-of-freedom absorber design ([11] and [1] or [10]) from the modal characteristics in Equation (6) and the modal mass ratio

$$
\mu_{i}=\frac{m_{a, i}}{m_{i}} .
$$

Moreover, Equations (6) and (7) give guidelines on where to place the absorber. Since the vibration reduction increases with the modal mass ratio, it is desirable to maximize this ratio. For a given absorber mass and a given forcing, the best placement is the one that maximizes the modal amplitude $\phi_{u, i}$ in the position at which the absorber is placed.

To mitigate multiple resonances, multiple LTVAs can be placed on the structure. The above procedure can be repeated $N$ times to tune the characteristics of $N$ vibration absorbers. This would result in a reduced amplitude around the $N$ targeted resonances, with ideally $N$ pairs of equal peaks.

Because the truncation of the harmonic response in Equation (5) involves neglecting several terms due to the non-resonant modes and because the interactions between the LTVAs are not properly taken into account by the aforementioned method, the frequency response function (FRF) will generally not exhibit perfectly equal peaks, as in the single-degree-of-freedom case. Numerical optimisation may be used to enforce equal peaks up to the desired accuracy. If two peaks related to mode $i$ are located at the frequencies $\omega_{i, 1}$ and $\omega_{i, 2}$ and if $H$ denotes the square modulus of the FRF for which equal peaks should be enforced, the following cost function can be used

$$
f\left(m_{a, 1}, c_{a, 1}, k_{a, 1}, \cdots, m_{a, N}, c_{a, N}, k_{a, N}\right)=\sum_{i=1}^{N}\left(H\left(\omega_{i, 1}\right)-H\left(\omega_{i, 2}\right)\right)^{2}
$$

Minimizing this cost function will result in a design with $N$ pairs of equal peaks. The peaks may be found by sampling the FRF, by using suitably initialised gradient ascent algorithms or by using $H_{\infty}$ norm computation algorithms [2] in a limited range of frequencies to properly locate all the peaks. The sensitivity of the cost function may be computed numerically through finite differences, or analytically. 


\section{Multimodal Nonlinear Tuned Vibration Absorber}

In this section, nonlinear elements are added to each absorbers to improve the vibration reduction in the nonlinear regime. It is assumed that the linear characteristics of the absorbers have been properly tuned so that the frequency response function of the controlled structure exhibits equal (or nearly equal) peaks in the linear regime. The functional form of the introduced nonlinear elements is chosen according to a principle of similarity, and their coefficients are computed by requiring that the nonlinear frequency response function exhibit equal peaks in the nonlinear regime.

The same formalism as Habib and Kerschen [7] is used in the following. The nonlinear equations of motion of the structure and of the nonlinear absorbers attached to it are given by

$$
\mathbf{M} \ddot{\mathbf{x}}+\mathbf{C} \dot{\mathbf{x}}+\mathbf{K} \mathbf{x}+k_{n l}\left(\mathbf{b}_{n l}(\mathbf{x})+\sum_{i=1}^{N} b_{n l, i} \mathbf{b}_{n l, i}(\mathbf{x})\right)=\mathbf{f}(t)
$$

where $\mathbf{b}_{n l}$ represents the nonlinearities in the primary structure, and $\mathbf{b}_{n l, i}$ are the nonlinearities in the absorbers. According to the principle of similarity [8], the latter are chosen to have the same mathematical form as the former. The remaining unknowns are the nonlinear coefficients of the absorbers, $b_{n l, i}$.

The equations of motion are normalised considering $\mathbf{y}=\mathbf{x} / f$, with $f$ being a forcing amplitude

$$
\mathbf{M} \ddot{\mathbf{y}}+\mathbf{C} \dot{\mathbf{y}}+\mathbf{K y}+\alpha\left(\mathbf{b}_{n l}(\mathbf{y})+\sum_{i=1}^{N} b_{n l, i} \mathbf{b}_{n l, i}(\mathbf{y})\right)=\frac{\mathbf{f}(t)}{f}
$$

$\alpha$ is a nonlinearity parameter that depends on both the nonlinearity and the forcing. For a polynomial nonlinear force of type $f_{n l}(x)=k_{n l} x^{p}$, it is given by

$$
\alpha=f^{p-1} k_{n l}
$$

This parameter can be seen as a measure of how the structural behaviour is affected by the nonlinearities. The solution of Equation (10) under a harmonic forcing can be approximated with a first-order harmonic balance (HB) method. Using

$$
\mathbf{y}=\mathbf{q}_{c} \cos (\omega t)+\mathbf{q}_{s} \sin (\omega t)
$$

and introducing $\mathbf{q}^{T}=\left[\mathbf{q}_{c}^{T}, \mathbf{q}_{s}^{T}\right]$, Equation (10) becomes

$$
\mathbf{W q}+\alpha\left(\mathbf{d}_{1, n l}(\mathbf{q})+\sum_{i=1}^{N} b_{n l, i} \mathbf{d}_{1, n l, i}(\mathbf{q})\right)=\mathbf{c}
$$

In Equation (13), the matrix $\mathbf{W}$ represents the linear dynamics, $\mathbf{c}$ is the forcing term, and the terms $\mathbf{d}_{1, n l}$ and $\mathbf{d}_{1, n l, i}$ represent the nonlinearities in the primary structure and in the $i^{t h}$ absorber, respectively. These vectors can be computed from 
q either analytically, or numerically with the alternating frequency/time-domain technique [3]. Thanks to the HB method, the set of nonlinear ordinary differential equations given in Equation (10) has been transformed into a set of nonlinear algebraic equations. To find an approximate solution, it is further assumed that the nonlinearity parameter $\alpha$ is small. Hence, the nonlinear response of the system may be expressed as a series expansion in terms of $\alpha$ as

$$
\mathbf{q}=\sum_{i=0}^{\infty} \alpha^{i} \mathbf{q}^{(i)}
$$

Inserting Equation (14) into (13) and equating coefficients of like powers of $\alpha$ up to first order leads to the following explicit relations :

$$
\begin{gathered}
\mathbf{q}^{(0)}=\mathbf{W}^{-1} \mathbf{c}, \\
\mathbf{q}^{(1)}=-\mathbf{W}^{-1}\left(\mathbf{d}_{1, n l}\left(\mathbf{q}^{(0)}\right)+\sum_{i=1}^{N} b_{n l, i} \mathbf{d}_{1, n l, i}\left(\mathbf{q}^{(0)}\right)\right)=\mathbf{q}_{n l}^{(1)}+\sum_{i=1}^{N} b_{n l, i} \mathbf{q}_{n l, i}^{(1)} .
\end{gathered}
$$

Equation (15) indicates that $\mathbf{q}^{(0)}$ represents the response of the system when no nonlinearity is present. Equation (16) shows that first-order terms $\mathbf{q}^{(1)}$ are generated by the nonlinear forces triggered by the zeroth-order motion. These terms can be separated into terms due to the nonlinearities inside the primary structure $\mathbf{q}_{n l}^{(1)}$ and terms due to the nonlinearities inside the $i^{t h}$ absorber $\mathbf{q}_{n l, i}^{(1)}$. The square of the frequency response function at a given degree of freedom is given by

$$
H=\left(\mathbf{q}_{c}\right)_{j}^{2}+\left(\mathbf{q}_{s}\right)_{j}^{2} .
$$

Inserting the solution of (15) and (16) into (17) and keeping only the first-order terms leads to

$$
\begin{aligned}
H=\left(\mathbf{q}_{c}^{(0)}\right)_{j}^{2}+\left(\mathbf{q}_{s}^{(0)}\right)_{j}^{2} & +\alpha\left[2\left(\mathbf{q}_{c}^{(0)}\right)_{j}\left(\mathbf{q}_{c, n l}^{(1)}\right)_{j}+2\left(\mathbf{q}_{s}^{(0)}\right)_{j}\left(\mathbf{q}_{s, n l}^{(1)}\right)_{j}\right] \\
& +\alpha\left[\sum_{i=1}^{N} b_{n l, i}\left[2\left(\mathbf{q}_{c}^{(0)}\right)_{j}\left(\mathbf{q}_{c, n l, i}^{(1)}\right)_{j}+2\left(\mathbf{q}_{s}^{(0)}\right)_{j}\left(\mathbf{q}_{s, n l, i}^{(1)}\right)_{j}\right]\right] . \\
& +\quad \frac{\alpha H_{n l}^{(1)}}{H^{(0)}} \\
& +
\end{aligned}
$$

In Equation (18), three types of term can be identified. The term $H^{(0)}$ stands for the frequency response function of the underlying linear structure. The term $\alpha H_{n l}^{(1)}$ is the modification brought by the (supposedly small) nonlinear forces generated by the linear motion in the nonlinearities of the primary structure. The term $\alpha b_{n l, i} H_{n l, i}^{(1)}$ is the modification brought by the (supposedly small) nonlinear forces generated by the 
linear motion in the nonlinearitiy $i$ of the absorbers. Thanks to the straightforward expansion, all these effects can be separated to first order in $\alpha$.

With Equation (18), it is now possible to impose $N$ conditions on the first-order nonlinear FRF. This yields a system of size $N \times N$, where the nonlinear coefficients $b_{n l, i}$ are the unknowns. Depending on the imposed conditions, this system can be linear and the nonlinear coefficients may or may not depend on the parameter $\alpha$.

\subsection{Equal peaks in the nonlinear regime}

A condition enforcing equal peaks in the nonlinear regime will now be derived. The linear absorbers are assumed to result in the equal-peak design, i. e. the peaks are equal in magnitude and located at frequencies $\omega_{i, 1}$ and $\omega_{i, 2}$ in the linear regime. The nonlinear effects cause a shift in these nonlinear peak frequencies, which are noted $\tilde{\omega}_{i, 1}$ and $\tilde{\omega}_{i, 2}$. Using a series development in $\alpha$ of the nonlinear frequency response, these nonlinear resonance frequencies are defined as

$$
\left.\frac{\partial H}{\partial \omega}\right|_{\tilde{\omega}_{i, j}}=\left.\sum_{n=0}^{\infty} \alpha^{n} \frac{\partial H^{(n)}}{\partial \omega}\right|_{\tilde{\omega}_{i, j}}=0
$$

They can be found by using a second series development in terms of the difference between the linear and nonlinear resonance frequencies $\Delta \omega_{i, j}=\tilde{\omega}_{i, j}-\omega_{i, j}$.

$$
\left.\frac{\partial H}{\partial \omega}\right|_{\tilde{\omega}_{i, j}}=\left.\sum_{n=0}^{\infty} \alpha^{n} \sum_{m=1}^{\infty} \frac{\left(\Delta \omega_{i, j}\right)^{m-1}}{(m-1) !} \frac{\partial^{m} H^{(n)}}{\partial \omega^{m}}\right|_{\omega_{i, j}}=0
$$

Keeping terms up to first order in $\Delta \omega_{i, j}$ and in $\alpha$ in the previous sum leads to

$$
\left.\frac{\partial H^{(0)}}{\partial \omega}\right|_{\omega_{i, j}}+\left.\Delta \omega_{i, j} \frac{\partial^{2} H^{(0)}}{\partial \omega^{2}}\right|_{\omega_{i, j}}+O\left(\Delta \omega_{i, j}\right)^{2}+\left.\alpha \frac{\partial H^{(1)}}{\partial \omega}\right|_{\omega_{i, j}}+O\left(\Delta \omega_{i, j} \alpha\right)=0
$$

Because $H^{(0)}$ is the linear frequency response function, the first term in Equation (21) vanishes. Hence, the nonlinear resonance frequency shift is of the order of $\alpha$. Now, the nonlinear frequency response at the nonlinear resonance frequency can be expressed as

$$
\left.H\right|_{\tilde{\omega}_{i, j}}=\left.\sum_{n=0}^{\infty} \alpha^{n} \sum_{m=0}^{\infty} \frac{\left(\Delta \omega_{i, j}\right)^{m}}{m !} \frac{\partial^{m} H^{(n)}}{\partial \omega^{m}}\right|_{\omega_{i, j}} .
$$

Evaluating Equation (22) at both nonlinear resonance frequencies, equating the two obtained nonlinear frequency response functions and keeping terms up to first order in $\alpha$ leads to 
$\left.H^{(0)}\right|_{\omega_{i, 1}}+\left.\Delta \omega_{i, 1} \frac{\partial H^{(0)}}{\partial \omega}\right|_{\omega_{i, 1}}+\left.\alpha H^{(1)}\right|_{\omega_{i, 1}}+O\left(\alpha^{2}\right)=\left.H^{(0)}\right|_{\omega_{i, 2}}+\left.\Delta \omega_{i, 2} \frac{\partial H^{(0)}}{\partial \omega}\right|_{\omega_{i, 2}}+\left.\alpha H^{(1)}\right|_{\omega_{i, 2}}+O\left(\alpha^{2}\right)$

Since equal peaks were assumed in the linear regime, the first terms in the left and right hand side of Equation (23) cancel out. Moreover, the second terms also vanish by definition of the linear resonance frequencies. Then, Equation (23) becomes independent of $\alpha$ and, using the contributions from the different nonlinearities highlighted by Equation (18), it can be written as

$$
\sum_{n=1}^{N} b_{n l, n}\left(H_{n l, n}^{(1)}\left(\omega_{i, 1}\right)-H_{n l, n}^{(1)}\left(\omega_{i, 2}\right)\right)=H_{n l}^{(1)}\left(\omega_{i, 2}\right)-H_{n l}^{(1)}\left(\omega_{i, 1}\right) .
$$

Interestingly, the nonlinear resonance frequency shift is not taken into account in the first-order frequency response function. Thus, the knowledge of the nonlinear resonance frequencies is not needed to design absorbers, at least to first order in $\alpha$. Enforcing Equation (24) for $i=1, \cdots, N$ yields the following linear system of size $N \times N$ :

$$
\left[\begin{array}{cccc}
\Delta_{\omega_{1}} H_{n l, 1}^{(1)} & \Delta_{\omega_{1}} H_{n l, 2}^{(1)} & \ldots & \Delta_{\omega_{1}} H_{n l, N}^{(1)} \\
\Delta_{\omega_{2}} H_{n l, 1}^{(1)} & \Delta_{\omega_{2}} H_{n l, 2}^{(1)} & \ldots & \vdots \\
\vdots & \vdots & \ddots & \vdots \\
\Delta_{\omega_{N}} H_{n l, 1}^{(1)} & \ldots & \ldots & \Delta_{\omega_{N}} H_{n l, N}^{(1)}
\end{array}\right]\left[\begin{array}{c}
b_{n l, 1} \\
b_{n l, 2} \\
\vdots \\
b_{n l, N}
\end{array}\right]=-\left[\begin{array}{c}
\Delta_{\omega_{1}} H_{n l}^{(1)} \\
\Delta_{\omega_{2}} H_{n l}^{(1)} \\
\vdots \\
\Delta_{\omega_{N}} H_{n l}^{(1)}
\end{array}\right],
$$

in which

$$
\Delta_{\omega_{i}} H=H\left(\omega_{i, 1}\right)-H\left(\omega_{i, 2}\right) .
$$

Equation (24) expresses that the (first-order) effects of all the nonlinearities is the same at $\omega_{i, 1}$ and $\omega_{i, 2}$. Therefore, the perfect equality between the amplitudes of the resonance peaks is not mandatory in practice. Peaks of approximately equal amplitude in the linear regime will remain approximately equal in the nonlinear regime.

Due to the series expansions limited to first order, this approach is a local approach, in the sense that the obtained results will gradually lose their validity with increasing $\alpha$, that is, as the forcing amplitude or the nonlinear coefficients become large. Nevertheless, it is expected that the nonlinear absorbers yield better result than their linear counterparts for small values of $\alpha$.

\section{Numerical example}

To demonstrate the efficiency of the proposed approach, a five-degree-of-freedom structure depicted in Figure 1 is studied, with numerical parameters given in Table 1. 
A cubic spring is attached to the first mass. A harmonic forcing is applied to this mass and the structural response is measured at this same point.

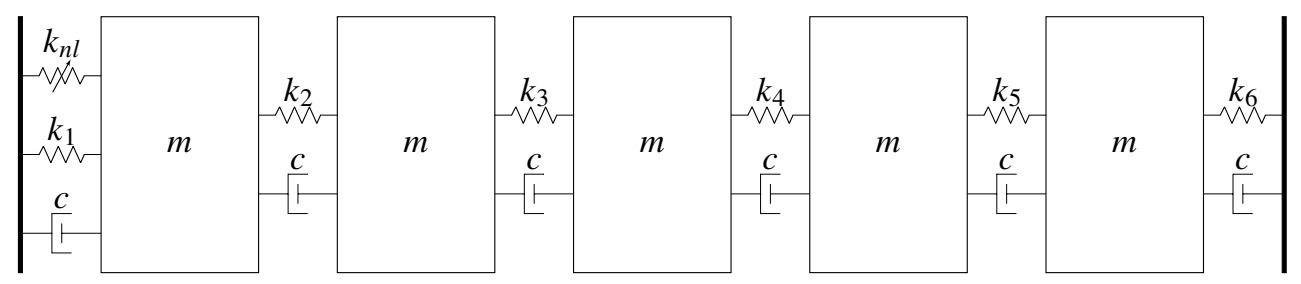

Fig. 1: Five-degree-of-freedom structure.

\begin{tabular}{|c|c|c|c|c|c|c|c|c|}
\hline$k_{1}$ & $k_{2}$ & $k_{3}$ & $k_{4}$ & $k_{5}$ & $k_{6}$ & $k_{n l}$ & $c$ & $m$ \\
\hline $1 \mathrm{~N} / \mathrm{m}$ & $1 \mathrm{~N} / \mathrm{m}$ & $0.1 \mathrm{~N} / \mathrm{m}$ & $1 \mathrm{~N} / \mathrm{m}$ & $1 \mathrm{~N} / \mathrm{m}$ & $1 \mathrm{~N} / \mathrm{m}$ & $1 \mathrm{~N} / \mathrm{m}^{3}$ & $0.03 \mathrm{Ns} / \mathrm{m}$ & $1 \mathrm{~kg}$ \\
\hline
\end{tabular}

Table 1: Numerical parameters of the five-degree-of-freedom structure.

Modes 1, 2 and 4 of the structure are targeted for vibration mitigation. The linear absorbers are placed at the maximum amplitude of the mode they are supposed to damp. Their parameters are optimised to obtain three pairs of equal peaks. The numerical values of the parameters are given in Table 2.

\begin{tabular}{|c|c|c|c|c|c|}
\hline Absorber & Location & $m_{a}$ & $c_{a}$ & $k_{a}$ & $k_{n l, a}$ \\
\hline Mode 1 & dof 3 & $0.05 \mathrm{~kg}$ & $3.88 \times 10^{-3} \mathrm{Ns} / \mathrm{m}$ & $1.16 \times 10^{-2} \mathrm{~N} / \mathrm{m}$ & $1.38 \times 10^{-5} \mathrm{~N} / \mathrm{m}^{3}$ \\
\hline Mode 2 & dof 2 & $0.05 \mathrm{~kg}$ & $6.03 \times 10^{-3} \mathrm{Ns} / \mathrm{m}$ & $2.19 \times 10^{-2} \mathrm{~N} / \mathrm{m}$ & $3.02 \times 10^{-4} \mathrm{~N} / \mathrm{m}^{3}$ \\
\hline Mode 4 & dof 1 & $0.05 \mathrm{~kg}$ & $1.66 \times 10^{-2} \mathrm{Ns} / \mathrm{m}$ & $1.27 \times 10^{-1} \mathrm{~N} / \mathrm{m}$ & $1.95 \times 10^{-3} \mathrm{~N} / \mathrm{m}^{3}$ \\
\hline
\end{tabular}

Table 2: Parameters of the absorbers.

Figure 2 shows the nonlinear frequency response functions (NFRFs) of the structure with linear and nonlinear absorbers for two values of the nonlinearity parameter $\alpha$. The NFRFs were computed with a continuation procedure coupled with a harmonic balance formalism [3] with five harmonics. For $\alpha=0.01$, the nonlinear effects are strong enough to detune the linear absorber of mode 2, whereas adding a nonlinear spring to that absorbers helps enforcing equal peaks. Mode 1 and 4 are less affected, namely due to their lower vibratory amplitude. When $\alpha$ is increased, mode 4 becomes affected. Equal peaks can be obtained thanks to the nonlinearities in the absorber for mode 4 . As for mode 2, the peaks can no longer be considered as equal, but the situation is still improved compared to that of the linear absorbers. Indeed, the maximum vibratory amplitude is lower when nonlinear absorbers are used.

Figure 3 shows how the amplitudes of the six peaks evolve with $\alpha$. When no nonlinear effect is present $(\alpha=0)$, the linear and nonlinear absorbers are equivalent. 


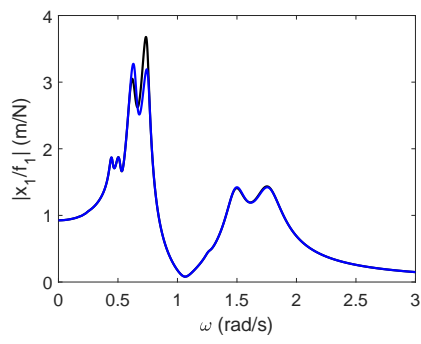

(a)

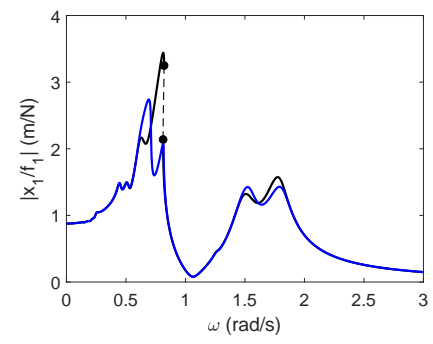

(b)

Fig. 2: NFRFs of the five-degree-of-freedom structure with linear (一: stable solution, - -: unstable solution, $\bullet$ : fold bifurcation) and nonlinear (-) absorbers:

$$
\alpha=0.01 \mathrm{~N}^{3} / \mathrm{m}^{3} \text { (a) and } \alpha=0.09 \mathrm{~N}^{3} / \mathrm{m}^{3} \text { (b). }
$$

As $\alpha$ increases, the two lines representing the pair of peaks associated to a particular mode diverges more quickly when no nonlinearity is used in the absorbers. Thanks to Equation (24), these lines are tangent at $\alpha=0$ when the nonlinearities are properly tuned, which slows down their divergence for small $\alpha$. This means that equal peaks are enforced over a broader amplitude range when using nonlinear absorbers. It is also clear that using nonlinear absorbers in this case leads to a lower maximum vibratory amplitude.



Fig. 3: Evolution of the peaks amplitudes with the nonlinearity parameter $\alpha$ : linear (一) and nonlinear (-) absorbers.

Going beyond that nonlinear regime can reveal more on the behaviour and the limitations of the proposed multimodal nonlinear tuned vibration absorber. The NFRFs of the structure in the strongly $(\alpha=0.36)$ and in the extremely $(\alpha=0.81)$ nonlinear regimes are shown in Figures 4 and 5, respectively. With nonlinear absorbers, the onset of quasiperiodic oscillations can be observed on mode two, with the appearance of a pair of Neimark-Sacker bifurcations. The amplitude of those vibrations can be computed through direct time integration of the equations of motion. The linear absorbers are progressively detuned, which results in higher vibratory amplitudes 
as $\alpha$ increases. With the nonlinear absorbers, a detached resonance curve (DRC) merges with the rightmost peak of mode 4 at $\alpha=0.518$, leading to the possibility of much higher vibration amplitudes. At this point, there is little difference of performance (in terms of maximal vibratory amplitude) between the linear and the nonlinear absorbers. Interestingly, the DRC of the nonlinear absorbers is connected to the NFRF through a branch of quasiperiodic solutions, which seems to lose its stability when the lower-amplitude periodic solutions become stable again, as the behaviour of the amplitude obtained with time integration seems to indicate.

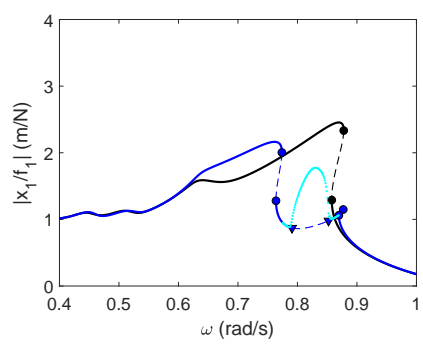

(a)

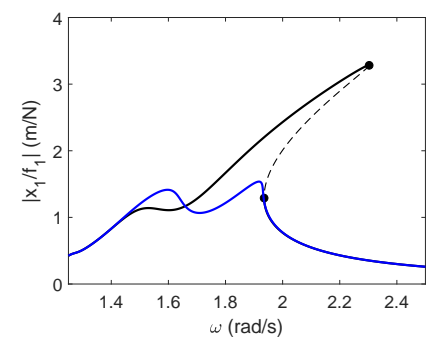

(b)

Fig. 4: NFRFs of the five-degree-of-freedom structure with linear (一: stable solution, - -: unstable solution, $\bullet$ : fold bifurcation) and nonlinear (一: stable solution, - -: unstable solution, $\bullet$ : fold bifurcation, $\boldsymbol{\nabla}$ : Neimark-Sacker bifurcation, • : time-integrated solution) absorbers at $\alpha=0.36 \mathrm{~N}^{3} / \mathrm{m}^{3}$ : close-up on modes 1 and 2

(a) and close-up on mode 4 (b).

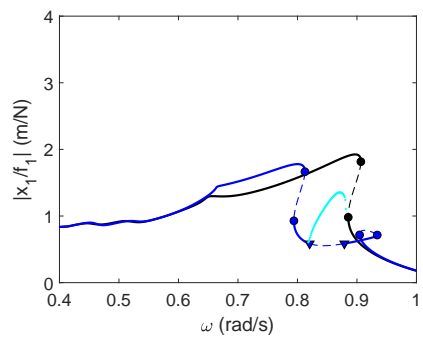

(a)

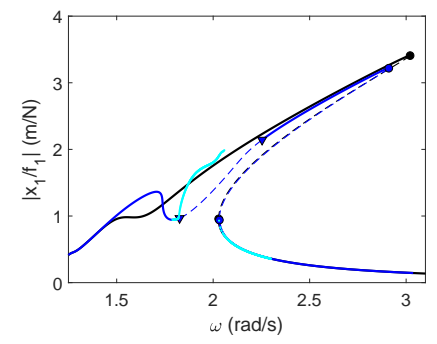

(b)

Fig. 5: NFRFs of the five-degree-of-freedom structure with linear (-: stable solution, - -: unstable solution, $\bullet$ : fold bifurcation) and nonlinear (-: stable solution, - -: unstable solution, $\bullet$ : fold bifurcation, $\boldsymbol{\nabla}$ : Neimark-Sacker bifurcation, • : time-integrated solution) absorbers at $\alpha=0.81 \mathrm{~N}^{3} / \mathrm{m}^{3}$ : close-up on modes 1 and 2

(a) and close-up on mode 4 (b). 


\section{Conclusion}

This paper proposed a tuning methodology for a multimodal nonlinear tuned vibration absorber. The linear characteristics of this absorber are first tuned. Based on a modal expansion of the frequency response function of the structure at the degree of freedom at which the absorbers are placed, an equivalent one-degree-of-freedom model is derived. The tuning rules of the absorber for a single-degree-of-freedom system can thus be used with multiple vibration absorbers to mitigate multiple resonances. A better equality between the peaks amplitudes can be obtained with the help of numerical optimisation. Nonlinearities are then added to the absorbers. Thanks to a principle of similarity, the functional form of these nonlinearities are chosen to be identical to those of the host structure. Their coefficients are determined by enforcing equal peaks in the nonlinear regime.

The example showed that the nonlinear absorbers placed on a nonlinear structure are more efficient than their linear counterpart. Not only are they able to enforce equal peaks over a broader amplitude range, but they also allow to obtain frequency response functions with lower maximal amplitudes than when using linear absorbers. The nonlinear absorbers trigger nonlinear phenomena, such as quasiperiodic oscillations and detached resonance curves. But even when these phenomena arise, the situation is at worse equivalent to that of the linear absorbers.

Future work may involve the use of a modal approach to simplify the computation of the nonlinear coefficients, therefore not requiring the full structural matrices.

Acknowledgements The authors would like to acknowledge the financial support of the SPW (WALInnov grant 1610122).

\section{References}

1. Brock, J.E.: A note on the damped vibration absorber. Trans. ASME, J. Appl. Mech. 13(4), A-284 (1946)

2. Bruinsma, N., Steinbuch, M.: A fast algorithm to compute the $h_{\infty}$-norm of a transfer function matrix. Systems \& Control Letters 14(4), 287-293 (1990)

3. Detroux, T., Renson, L., Masset, L., Kerschen, G.: The harmonic balance method for bifurcation analysis of large-scale nonlinear mechanical systems. Computer Methods in Applied Mechanics and Engineering 296, 18-38 (2015)

4. Elias, S., Matsagar, V.: Research developments in vibration control of structures using passive tuned mass dampers. Annual Reviews in Control 44, 129-156 (2017). DOI 10.1016/j.arcontrol.2017.09.015

5. Frahm, H.: Device for damping vibrations of bodies. (1911). US Patent 989,958

6. Géradin, M., Rixen, D.J.: Mechanical vibrations: theory and application to structural dynamics. John Wiley \& Sons (2014)

7. Habib, G., Grappasonni, C., Kerschen, G.: Passive linearization of nonlinear resonances. Journal of Applied Physics 120(4), 4-9 (2016). DOI 10.1063/1.4959814

8. Habib, G., Kerschen, G.: A principle of similarity for nonlinear vibration absorbers. Physica D: Nonlinear Phenomena 332, 1-8 (2016) 
9. Krenk, S., Høgsberg, J.: Tuned resonant mass or inerter-based absorbers: Unified calibration with quasi-dynamic flexibility and inertia correction. Proceedings of the Royal Society A: Mathematical, Physical and Engineering Sciences 472(2185) (2016). DOI 10.1098/rspa.2015.0718

10. Nishihara, O., Asami, T.: Closed-form solutions to the exact optimizations of dynamic vibration absorbers (minimizations of the maximum amplitude magnification factors). Journal of vibration and acoustics 124(4), 576-582 (2002)

11. Ormondroyd, J.: The theory of the dynamic vibration absorber. Trans., ASME, Applied Mechanics 50, 9-22 (1928)

12. Ozer, M.B., Royston, T.J.: Extending den hartog's vibration absorber technique to multidegree-of-freedom systems. Journal of Vibration and Acoustics 127(4), 341-350 (2005) 